\title{
Patterns of alcohol consumption after liver transplantation
}

\author{
H Tang, R Boulton, B Gunson, S Hubscher, J Neuberger
}

\begin{abstract}
Background-Uncertainty exists about the extent and consequences of a return to alcohol consumption after liver transplantation for alcoholic liver disease (ALD). Aims-To determine the prevalence and consequences of alcohol consumption in patients transplanted for ALD.

Methods-A retrospective case controlled study of all patients transplanted for ALD at the Queen Elizabeth Hospital, Birmingham, between 1987 and 1996.

Results-Seventy patients with ALD were transplanted, of which 59 survived more than three months; 56 were interviewed. Twenty eight had consumed some alcohol after transplantation; for the nine "heavy drinkers" (HD), the median time to resumption of alcohol intake was six months and for the 19 "moderate drinkers" (MD) it was eight months. There was no significant difference in episodes of acute rejection or compliance with medication between those who were abstinent, MD, or HD. Histological evidence of liver injury was common in ALD patients who had returned to drink. Mild fatty change was found in 1/11 biopsy specimens from abstinent patients but moderate to severe fatty change and ballooned hepatocytes were seen in 3/5 MD and 2/5 HD specimens. Two HD patients had early fibrosis. One HD patient has died of alcohol related complications.

Conclusions-Moderate to heavy alcohol consumption occurs in patients transplanted for ALD. Patient recall of abstinence advice is unreliable, and patients return to alcohol mainly within the first year after liver transplantation. Return to alcohol consumption after liver transplantation is associated with rapid development of histological liver injury including fibrosis.
\end{abstract}

(Gut 1998;43:140-145)

Keywords: alcohol consumption; liver transplantation

Liver Unit, Queen

Elizabeth Hospital, Birmingham

B15 2TH, UK

H Tang

$\mathrm{R}$ Boulton

B Gunson

S Hubscher

J Neuberger

Correspondence to:

Dr Neuberger.

Accepted for publication 19 January 1998 cubsequently reported simi results. $^{3-16}$ Outcome, in these studies, has equalled or bettered the results of liver transplantation in patients with non-alcoholic cirrhosis. $^{3-91115}$ Partly as a consequence of these encouraging results increasing numbers of patients have been transplanted for ALD in recent years and it has become the best therapeutic option in end stage disease. ${ }^{17}$ Nonetheless, the role of orthotopic liver transplantation in patients with end stage ALD remains controversial.

There have been concerns over the increasing use of a limited supply of donor livers to treat a condition which is self inflicted, as it inevitably denies patients with non-alcoholic liver disease the chance of transplantation. ${ }^{18}$ Knowledge that a liver may be allocated to a patient with ALD may increase reluctance of relatives to give consent for organ donation. However, although the liver injury is self inflicted, many clinicians believe the liver damage is the consequence of a disease-alcohol dependence ${ }^{19}$ - and it is unjust to discriminate against one disease in favour of another. Alcohol dependence has clearly defined diagnostic criteria $^{20}$ and is characterised by notable preoccupation with drinking and impaired control and an associated distortion in thinking that leads to a denial of the relation between alcohol consumption and its adverse consequences. Not all patients with ALD suffer from alcohol dependence; some have a condition defined as alcohol abuse and some defy psychiatric diagnosis. ${ }^{21}$ In others concomitant viral or metabolic disease (for example, haemochromatosis) increases susceptibility to injury.

Another concern about the use of transplantation for patients with ALD has been the high rate of recidivism among alcoholic patients following detoxification and rehabilitation. ${ }^{22}$ The incidence of recidivism in patients transplanted for ALD is uncertain, with a reported incidence ranging from 0 to $95 \% .^{3-16}{ }^{23-27}$ European centres tend to report higher rates. ${ }^{3-5} 89^{13-16}{ }^{28} \mathrm{~A}$ lack of consensus on the definition of recidivism has complicated the comparison between results reported by different centres.

It has been presumed that patients transplanted for ALD subsequently resume a harmful pattern of alcohol consumption that would have important implications including noncompliance with immunosuppression and consequent rejection, and alcoholic injury to the allograft and non-hepatic tissues. The purpose of this study was to assess post-transplant alcohol consumption and review the outcome of patients transplanted for ALD at the Queen Elizabeth Hospital, Birmingham.

\section{Methods}

The study was a retrospective case controlled study of all patients transplanted for end stage alcoholic liver disease between January 1987 
and May 1996. Patients were selected for transplantation if the anticipated survival (in the absence of transplantation) was estimated to be less than one year and there was no evidence of other end organ damage, such as cardiomyopathy or cerebral damage. All patients were evaluated by a psychiatrist and accepted for transplantation if it was believed that the patient was not alcohol dependent and would be unlikely to return to alcohol consumption. It was the policy of the unit that patients should be abstinent for six months prior to listing although it was recognised that for a small group of patients, the hepatic reserve did not allow for such an interval. Postoperative immunosuppression was prednisolone, azathioprine, and cyclosporin as described elsewhere. ${ }^{29}$ Biochemical and survival data were retrieved from a computer database and by review of the patients' case records. Liver biopsies were performed as dictated by clinical circumstances - usually to investigate unexplained derangement of the liver biochemistry and/or suspicion of rejection.

Patients who had survived more than three months after transplantation were contacted. A group of age and sex matched patients transplanted during the same period for chronic non-alcoholic end stage liver disease was selected as a comparison group and these patients were also interviewed. Patients were asked in a confidential, structured interview about post-transplant alcohol consumption, time of return to alcohol consumption, recollection of advice received regarding posttransplant alcohol consumption, and employment history. For this study alcohol consumption was stratified into three groups: abstinent, no alcohol consumption; moderate, defined as less than $200 \mathrm{~g}$ (20 units) per week; and heavy drinking, defined as more than 200 $\mathrm{g}$ per week. The confidentiality of the enquiry was stressed.

DIAGNOSTIC CRITERIA

The preoperative diagnosis of ALD was based on a history of excessive and persistent alcohol consumption $^{30}$ together with compatible clinical and laboratory findings and liver biopsy results. The diagnosis was confirmed by histological assessment of the explanted liver.

Psychiatric assessment pretransplantation

To address patients' suitability for transplantation all were interviewed by an experienced multidisciplinary team, including a psychiatrist and a specialist social worker ${ }^{31}$ using a structured psychosocial assessment. Patients transplanted after 1990 all fulfilled DSM-III-R criteria for alcohol dependence or alcohol abuse. ${ }^{20}$ All patients were instructed to continue abstinence after liver transplantation, but no formal "contract of abstinence" was made.

Physical assessment

Patients were considered for transplantation if their physical prognosis was poor despite abstinence and there was no significant alcohol induced extrahepatic end organ damage.
STATISTICAL ANALYSIS

Fisher's exact test was used to compare differences between patient groups and the MannWhitney test was used to determine differences in relapse times between heavy and moderate drinkers. Survival analysis was determined using the Kaplan-Meier method.

\section{Results}

PRETRANSPLANTATION CHARACTERISTICS

Seventy one patients with a primary preoperative diagnosis of ALD underwent orthotopic liver transplantation between January 1987 and May 1996. Of these 69 were performed after 1990 and accounted for $7.4 \%$ of all adult liver transplants performed during this period. There were 64 men $(90 \%)$ and seven women $(10 \%)$; median age at the time of transplantation was 48 years (range 29-68). Immediately prior to transplantation, $7 \%$ were classified as Pugh grade A, $23 \%$ as grade $\mathrm{B}$, and $70 \%$ as grade C. Only two patients had antibodies to hepatitis $\mathrm{C}$ virus; the histology of the explanted liver showed evidence of inactive cirrhosis. Recurrent hepatitis C virus infection resulted in cirrhosis in the allograft of one patient. The median period of abstinence prior to orthotopic liver transplantation was 12 months (range 0-96 months); $76 \%$ of patients were abstinent for at least six months prior to transplantation.

\section{HISTOLOGY}

All the explanted livers were cirrhotic; alcoholic hepatitis was present in only one liver and another patient had coincidental hepatocellular carcinoma.

SURVIVAL ANALYSIS

The one and two year actuarial survival for patients undergoing liver transplantation for ALD was $82.8 \%$ and $80.0 \%$ respectively. The median time to death or census was 24 months (range 0-84 months). Twelve patients died. Two deaths occurred intraoperatively (one from cardiac arrest and one from cardiac failure) and eight deaths occurred in the early postoperative period (due to sepsis in five and haemorrhage in three). Two patients died three or more months after transplantation. One patient had resumed heavy alcohol consumption and died from pneumonia and heart failure; the other died from respiratory failure.

\section{ALCOHOL INTAKE POST-TRANSPLANTATION}

Clinical and laboratory data were available for the 59 patients surviving more than three months post-transplantation at the beginning of the study. Patients were questioned in a confidential interview; information could not be obtained from three patients (two had returned overseas and one could not be contacted).

Thirty one patients $(52 \%)$ claimed they were abstinent. Nineteen patients (32\%) said they only consumed alcohol on special occasions, and this never exceeded $200 \mathrm{~g}$ of alcohol per week; these were classified as moderate drinkers. Only two moderate drinkers consumed more than $10 \mathrm{~g}$ per day on average. Nine patients $(15 \%)$ were classified as heavy 
Table 1 Pretransplant abstinence and alcohol consumption post-transplant

\begin{tabular}{lll}
\hline \multirow{2}{*}{$\begin{array}{l}\text { Alcohol consumption } \\
\text { post-transplant }\end{array}$} & \multicolumn{2}{c}{ Pretransplant abstinence (months) } \\
\cline { 2 - 3 } & Median & Range \\
\hline Abstinent & 12 & $0-96$ \\
Moderate & 15 & $5-60$ \\
Heavy & 8 & $6-18$ \\
\hline
\end{tabular}

drinkers. No significant differences in the length of pretransplant abstinence was found between the abstinent and moderate drinkers and all post-transplant heavy drinkers claimed abstinence of at least six months preceding transplantation (table 1). Table 2 shows the outcome of the nine heavy drinkers. All adopted/resumed drinking within six months of transplantation. Moderate drinkers resumed alcohol consumption at a significantly slower rate; the median interval to relapse was six months for heavy drinkers and eight months for moderate drinkers $(\mathrm{p}=0.025)$ (fig 1$)$.

Alcohol consumption was assessed in the age and sex matched non-ALD transplanted comparison group. Of 39 patients interviewed, 23 (59\%) said they consumed alcohol after their transplant, but none were heavy drinkers.

PATIENT RECOLLECTION OF MEDICAL ADVICE ON ALCOHOL CONSUMPTION

Of the 56 ALD patients who were interviewed, $33(59 \%)$ could not recollect receiving advice on post-transplant alcohol consumption during their preoperative assessment. Ten (18\%) patients (correctly) recollected advice to abstain completely and $13(23 \%)$ claimed they were advised that drinking in moderation was acceptable (table 3 ). In the comparison group, $40 \%$ recalled being given advice on posttransplant alcohol consumption; they all (correctly) recalled being instructed that their health would not be jeopardised by drinking alcohol in moderation.

COMPLIANCE WITH IMMUNOSUPPRESSION

There were no significant differences in the incidence of suboptimal cyclosporin concentrations (defined as a trough value of less than $70 \mathrm{ng} / \mathrm{ml}$ ) between abstinent patients, moderate drinkers, and heavy drinkers $(6.1 \%, 0.6 \%$, and $6.5 \%$, respectively).

POST-TRANSPLANTATION LIVER HISTOLOGY For the 59 ALD patients the cumulative number of acute rejection episodes per patient

Table 2 Outcome in the nine heavy drinkers after transplantation

\begin{tabular}{|c|c|c|c|}
\hline $\begin{array}{l}\text { Patient } \\
\text { age/sex }\end{array}$ & Employed & $\begin{array}{l}\text { Follow up } \\
\text { (months) }\end{array}$ & Current status \\
\hline $40 / \mathrm{M}$ & No & 26 & $\begin{array}{l}\text { Frequent non-attendance. Died after } 26 \\
\text { months (heart failure, pneumonia and } \\
\text { alcoholic liver injury) }\end{array}$ \\
\hline $46 / \mathrm{M}$ & Yes & 56 & Alcohol 200-300 g/week. Pancreatitis \\
\hline $54 / \mathrm{M}$ & No & 18 & $\begin{array}{l}\text { Alcohol } 500 \mathrm{~g} / \text { week. Required inpatient } \\
\text { detoxification. Recent divorce }\end{array}$ \\
\hline $47 / \mathrm{F}$ & No & 24 & Alcohol $280 \mathrm{~g} /$ week \\
\hline $42 / \mathrm{M}$ & No & 24 & $\begin{array}{l}\text { Frequent non-attendance. Required } \\
\text { inpatient detoxification }\end{array}$ \\
\hline $48 / \mathrm{M}$ & No & 8 & $\begin{array}{l}\text { Alcohol } 400 \mathrm{~g} / \text { week. Admissions with } \\
\text { non-specific abdominal pain; depression }\end{array}$ \\
\hline $40 / \mathrm{M}$ & Yes & 33 & Alcohol $300 \mathrm{~g} /$ week \\
\hline $67 / \mathrm{M}$ & Retired & 27 & Alcohol >300 g/week \\
\hline $46 / \mathrm{M}$ & Yes & 33 & Alcohol $30-35 \mathrm{~g} /$ week \\
\hline
\end{tabular}

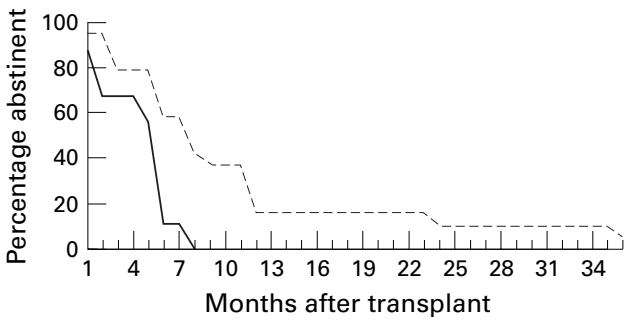

Figure 1 Recidivism post-transplantation for ALD.

was $0.46(27 / 59)$. Only two of these episodes occurred after 30 days (one in an abstinent patient and one in a moderate drinker) and both responded to high dose corticosteroid treatment. One patient has developed chronic rejection. The prevalence of chronic rejection in patients transplanted at our centre is $5.2 \% .^{31}$

Twenty one patients had a total of 46 follow up liver biopsies and were segregated according to their claimed alcohol consumption. Fatty change was found in $1 / 11$ abstinent patients, $3 / 5$ moderate drinkers, and $2 / 5$ heavy drinkers. Changes were more noticeable in the moderate and heavy drinkers, including early perivenular fibrosis with fatty change in the two heavy drinkers (table 4). In the comparison group, 19 patients had 43 follow up biopsies; fatty change was seen in four patients (two were abstinent, and two consumed 1-2 units of alcohol per week).

\section{EMPLOYMENT}

Before liver transplantation five patients with ALD were employed and at the time of the study 31 patients $(52 \%)$ had returned to either full time or part time paid employment (compared with $47.5 \%$ in the control group). Ten patients with ALD were retired or performing domestic duties. Seven of 31 abstinent patients compared with six of 19 moderate drinkers (NS) and five of nine heavy drinkers (NS) were unemployed. Of the unemployed abstinent patients, three of seven were less than 12 months post-transplantation compared with

Table 3 Reported advice on alcohol consumption

\begin{tabular}{llll}
\hline & \multicolumn{3}{l}{ Level of alcohol consumption } \\
\cline { 2 - 4 } Reported advice given & $\begin{array}{l}\text { Abstinent } \\
(n=31)\end{array}$ & $\begin{array}{l}\text { Moderate } \\
(n=19)\end{array}$ & $\begin{array}{l}\text { Heavy } \\
(n=9)\end{array}$ \\
\hline No advice & 24 & 3 & 6 \\
Abstain & 5 & 4 & 1 \\
Moderation & 1 & 12 & 0 \\
No data & 1 & 0 & 2
\end{tabular}

Table 4 Histological diagnosis post-transplant for ALD

\begin{tabular}{lrrr}
\hline & Abstinent & Moderate & Heavy \\
\hline Median follow up (days) & 550 & 414 & 362 \\
Range (days) & $126-1376$ & $107-1485$ & $148-427$ \\
Total number of biopsies & 24 & 14 & 9 \\
Total number of patients & 11 & 5 & 5 \\
Total patients with fatty & $1 / 11$ & $3 / 5$ & $2 / 5$ \\
$\quad$ change & & & \\
Mild fatty change & 1 & 0 & 0 \\
Moderate/severe fatty & 0 & 3 & 2 \\
$\quad$ change & 0 & 3 & 2 \\
Ballooning of hepatocytes & 1 & 0 & 0 \\
Mild portal infiltrate & 0 & 3 & 2 \\
Moderate portal infiltrate & 0 & 0 & 2 \\
Fibrosis & & & \\
\hline
\end{tabular}


Table 5 Published studies on alcohol consumption post-transplantation for ALD

\begin{tabular}{|c|c|c|c|c|}
\hline Reference & $\begin{array}{l}\text { No of } \\
\text { patients }\end{array}$ & $\begin{array}{l}\text { Follow up } \\
\text { (months) }\end{array}$ & Recidivism (\%) & Method \\
\hline Howard et $a l^{14}$ & 20 & 34 & 95 & Interview \\
\hline Gerhardt et $a l^{17}$ & 67 & $36-96$ & 49 & Interview \\
\hline Doffoel et a $l^{8}$ & 57 & NS & 33 & NS \\
\hline Berlakovich et al $l^{13}$ & 44 & 78 & 31 & Clinic \\
\hline Raakow et $a l^{15}$ & 78 & 25 & 22 & NS \\
\hline Bird et $a l^{4}$ & 18 & NS & 22 & Clinic/questionnaire \\
\hline Orsano et al ${ }^{12}$ & 37 & 21 & 19 & Questionnaire \\
\hline Foster et a $\beta^{4}$ & 63 & 49 & 21 & Interview \\
\hline Gish et al & 29 & 24 & 21 & Collaborative evidence \\
\hline Weisner et $\mathrm{l}^{18}$ & 139 & NS & 16 & Questionnaire \\
\hline Goldstein $e t a l^{10}$ & 37 & 27.5 & 14 & Retrospective \\
\hline Knechtle et $a \bar{l}$ & 32 & NS & 13 & Interview \\
\hline Krom et $a l^{11}$ & 30 & NS & 13 & NS \\
\hline Anand $e t a l^{9}$ & 39 & 25 & 13 & Retrospective \\
\hline Reeck et $a l^{16}$ & 52 & NS & 12 & NS \\
\hline Kumar $e t a l^{3}$ & 73 & 25 & 11.5 & Telephone survey \\
\hline Lucey et $a l^{6}$ & 45 & NS & 11 & Telephone survey/clinic \\
\hline Bharadhwaj et al ${ }^{19}$ & 46 & $>12$ & 11 & Interview \\
\hline Pageaux et al $\varphi^{9}$ & 22 & 12 & 9 & Clinic \\
\hline Stevens et $a l^{18}$ & 10 & 24 & 0 & NS \\
\hline
\end{tabular}

NS, not stated.

one of six and one of five moderate and heavy drinkers respectively.

\section{Discussion}

Although this report describes only a small, selected group of patients with alcoholic liver disease, about half the patients transplanted for alcoholic liver disease at our centre admitted consuming alcohol after liver transplantation. Furthermore, those that consistently consumed significant amounts did so within one year of their operation. Both these findings are at variance with reports from other centres.

Accurate assessment of alcohol consumption post-transplantation is beset with difficulty. In a recent European survey only seven of 16 centres had even instituted routine screening for a return to alcohol consumption in patients transplanted for ALD. ${ }^{28}$ Screening methods varied, ranging from rigorous interviews and questionnaires to brief outpatient questioning about drinking habits. One problem is the lack of consensus as to the best prospective measurement of a return to drinking. Random checks of blood and urine for alcohol require formal evaluation and review of patient case notes with collaborative evidence from other medical staff which is often inconsistent and incomplete. Metabolic indices such as carbohydrate specific transferrin have been shown to lack specificity ${ }^{32}$ and interpretation of histological and biochemical changes in the posttransplant liver is difficult and not specific.

Our data on recidivism were based on a post-transplant follow up interview. We suspect our patients were more willing to admit to post-transplant alcohol consumption because we stressed that the information was strictly confidential and that it would be withheld from their case notes. This may also explain why a higher level of alcohol consumption was detected in this study compared with a previous study from this centre which relied on patient case notes and collaborative evidence to detect recidivism. ${ }^{33}$

These data indicate that alcohol consumption is common among patients transplanted for ALD. Only one other centre has reported a higher prevalence of post-transplant alcohol consumption in ALD patients than we report here. ${ }^{14}$ That was a study of a smaller number of patients and although rigorous and well conducted, only 20 of the 54 patients transplanted for ALD were evaluated. Several factors contribute to the high prevalence of alcohol consumption we describe. Firstly, most of the moderate drinkers incorrectly claimed they received advice that this was acceptable (table 3); this may be their justification for their drinking pattern. Secondly, no specific "contract" of lifelong abstinence was made although patients transplanted for ALD were advised to be abstinent. Thirdly, the emphasis on the confidential nature of the structured interview may have encouraged greater honesty from patients in their responses. This is supported by a comparison of information collection techniques and recidivism rates (table 5). Indeed, in two reports from the same centre, the estimate of recidivism was $22 \%$ when patient notes were analysed $^{4}$ but $95 \%$ when estimated using a structured interview. ${ }^{14}$ Finally, of course, our preoperative selection process may be inadequate and this is currently being assessed prospectively.

In this study, the duration of abstinence prior to transplantation did not predict posttransplantation recidivism. Evidence from other centres is mixed, ${ }^{34}$ with support for $^{34811121516}$ and against ${ }^{1423}$ a correlation between duration of pretransplant abstinence and subsequent post-transplant recidivism. This has important implications for severely ill patients with ALD, as they have the most to gain from transplantation, ${ }^{26}{ }^{29}$ but have insufficient reserve to wait long for a graft. Our data suggest that the emphasis should shift to continuing post-transplantation treatment of the patient's primary addictive illness rather than pretransplantation abstinence.

The time to relapse into alcohol consumption at our centre was rapid, occurring within a year of operation when patients are under close follow up. Resumption of alcohol consumption was most rapid in patients who had returned to heavy drinking; all of the heavy drinkers had resumed alcohol consumption within six months of transplantation. An early return to alcohol consumption was also seen in the moderate drinkers (after a median of eight months). Other studies have suggested that the return to alcohol consumption occurs much later. $^{1423} 27$ Our data suggest that the risk of returning to alcohol consumption is greatest within the first year post-transplant, supporting the contention that alcohol dependence is a disease, ${ }^{19}$ with no reliable cure. It is also a salutary warning for the physician: there is no "safe period" when the patient is not at risk of returning to alcohol consumption. Indeed support of the ALD patient should start before the patient leaves the hospital.

Although a significant number of patients did return to alcohol consumption, over half the patients claimed to consume no alcohol. Patients' recollection of the advice they were given to remain abstinent postoperatively did not influence their postoperative drinking pattern. Over two thirds of the group claiming no 
advice to abstain was ever given remained abstinent (table 3). This is both unexpected and encouraging as it suggests that a large group of highly motivated patients are able to abstain after transplantation for ALD despite poor recollection of the information they are given. These data also illustrate that communication of alcohol consumption advice is imperfectly recalled and given the relatively short follow up period of 24 months, little can be assumed about longer term abstinence.

How important is abstinence for patients transplanted for ALD? There were no significant differences in the number of episodes of rejection, or compliance with cyclosporin therapy between the abstinent, moderate, or heavy drinking patients but numbers are small. Histological evidence of alcohol induced liver injury was apparent in the liver biopsy specimens of alcohol consuming patients. Fatty change was seen more frequently in specimens from patients who consumed alcohol compared with only one of the 11 specimens from abstinent patients. The importance of mild fatty infiltration is uncertain: it was also noted in four of the biopsy specimens from patients in the non-ALD comparison group (two were abstinent and two admitted to drinking alcohol in moderation), and numbers of biopsy specimens examined is small. However, more significant fatty change-graded as moderate or severe and associated with ballooning of hepatocytes-was noted in the biopsy specimens of patients admitting to moderate or heavy alcohol consumption. Pericellular fibrosis was present in two biopsy specimens from patients with heavy alcohol intake. There are only limited data on the influence of alcohol consumption on post-transplantation liver histology, but our results support previous findings suggesting early progression of alcoholic injury with cirrhosis occurring from as early as six to 21 months. ${ }^{32}{ }^{35}{ }^{36}$ As patients in this series did not all have a routine, protocol annual biopsy, it is difficult to draw firm conclusions about the histological findings in this small number of patients.

Employment, as an index of social reintegration, is another measure of the outcome of transplantation in patients with ALD. It has limitations, especially as these patients often face social discriminatory hurdles that are as important as their physical health in preventing them from returning to work. About one third of patients transplanted for ALD and not yet working had had their operation less than 12 months before so their capacity for future employment is not clear. Despite this, $52 \%$ of the patients transplanted for ALD were in paid employment, a figure comparable with that in the control group (48\%).

Although undergoing liver transplantation can be viewed as a traumatic learning experience for the patient with ALD, it is clearly insufficient to promote abstinence and the risk of returning to alcohol consumption remains. In contrast to other studies, our experience indicates that many patients resume alcohol consumption very early after transplantation. The consequences for heavy drinkers are clear, but the risks of more moderate consumption will only be determined by long term prospective follow up of all patients, including careful monitoring of alcohol consumption and regular planned liver biopsies. Physicians should be aware that the experience of liver transplantation is not a disincentive to the patient with ALD to return to alcohol consumption. Our evidence indicates that patients resume alcohol consumption early after transplantation and their grafts can be injured. Advice needs to be continually repeated. Based on these observations, we have altered our policy of assessing patients with ALD for transplantation: we are using more intensive assessment, looking for patient acceptance of their alcohol excess, alternative strategies to deal with those factors leading the person to abuse alcohol, and strong social support. We are also asking the patients to acknowledge formally the expectation that they will abstain from alcohol and are providing more support in the first postoperative year.

We are grateful to the surgeons (Professor P McMaster, Mr J Buckels, and Mr A Mayer), physicians (Professor E Elias, Professor D Adams, and Dr D Mutimer), Dr A White (consultant psychiatrist), and the medical, nursing, and other colleagues involved in the care of these patients for allowing us to report this series. Some of the data in this paper have been published in abstract form (Tang H, Gunson BK, Boulton RA, Neuberger JM. Liver transplantation (LT) for alcoholic liver disease (ALD): lessons for patient and the physician. Gastroenterology 1997;122:A1396).

1 Consensus Conference on Liver Transplantation. $7 A M A$ 1983;250:2961-4.

2 Starzl TE, Van Theil D, Tzakis, et al. Orthotopic liver transplantation for alcoholic cirrhosis. FAMA 1988;260:2542-4. 3 Kumar S, Stauber RE, Gavalar JS, et al. Orthotopic liver transplantation for alcoholic liver disease. Hepatology 1990; 11:159-64.

4 Bird GL, O'Grady JG, Harvey FA, et al. Liver transplantation in patients with alcoholic cirrhosis: selection criteria and rates of survival and relapse. BMF 1990;301:15-17.

5 Knechtle SJ, Fleming MF, Barry KL, et al. Liver transplantation for alcoholic liver disease. Surgery 1992;112:694703.

6 Lucey MR, Merion RM, Henley KS, et al. Selection for and outcome of liver transplantation in alcoholic liver disease. Gastroenterology 1992;102:1736-41.

7 Gish RG, Lee AH, Keeffe EB, et al. Liver transplantation for Gish RG, Lee AH, Keeffe EB, et al. Liver transplantation for
patients with alcoholism and end-stage liver disease. Am $\mathcal{F}$ pastroenterol 1993;88:1337-42.

8 Doffoel M, Fratte S, Vanlemmens C, et al. Results of liver transplantation (LT) in 75 French patients with alcoholic cirrhosis (AC). Comparison with a nonalcoholic cirrhotic group [abstract]. Hepatology 1992;16:50A.

9 Pageaux GP, Souche P, Perney B, et al. Results and cost of orthotopic liver transplantation for alcoholic cirrhosis. Transplant Proc 1993;25:1135-6.

10 Goldstein R, Tripp L, Clemmons J, et al. Liver transplantaion for alcoholic cirrhosis-do they mix? Transplant Proc 1993;25:1131-2.

11 Krom RAF. Liver transplantation and alcohol: who should get transplants? Hepatology 1994;20:28S-32S

12 Orsono RW, Ascher NL, Avery M, et al. Predicting recidivism after orthotopic liver transplantation for alcoholic liver disease. Hepatology 1994;20:105-10.

13 Berlakovich GA, Steininger R, Herbst F, et al. Efficacy of Berlakovich GA, Steininger R, Herbst F, et al. Efficacy of
liver transplantation for alcoholic cirrhosis with respect to liver transplantation for alcoholic cirrhosis with respect to

14 Howard L, Fahy T, Wong P, et al. Psychiatric outcome in alcoholic transplant patients. $Q \mathcal{F}$ Med 1994;87:731-6.

15 Raakow R, Langrehr R, Lohmann $M$, et al. Is orthotopic liver transplantation for end-stage alcoholic cirrhosis justified? Transplant Proc 1995;27:1241-2.

16 Reeck UH, Egerer G, Theilmann C, et al. Liver transplantation for alcoholic cirrhosis: an 8 year follow up study [abstract]. Hepatology 1995;22:479A.

17 Poynard T, Barthelemy P, Fratte S, et al. Evaluation of efficacy of liver transplantation in alcoholic cirrhosis by a case control study and simulated controls. Lancet 1994;344: 502-7.

18 Moss AH, Siegler M. Should alcoholics compete equally for liver transplantation? $\mathcal{F A M A}$ 1991;265:1295-8.

19 Morse RM, Flavin DK. The definition of alcoholism. fAMA 1992;268:1012-14.

20 American Psychiatric Association. The diagnostic and statistical manual, 3rd edn, revised. Washington, DC: American Psychiatric Association, 1987. 
21 Beresford TP. Overt and covert alcoholism. In: Lucey MR, Merion RM, Beresford TP, eds. Liver transplantation and the alcoholic patient: medical, surgical and psychcosocial issues. Cambridge: Cambridge University Press, 1994.

22 Moos RM, Finney JW, Conkrite RO. Alcoholism treatment. New York: Oxford University Press, 1990.

23 Gerhardt TC, Goldstein RM, Ursschel L, et al. Alcohol use following liver transplantation for alcoholic cirrhosis. Transplantation 1996;62:1060-3.

24 Stevens L, Piper J, Emond T, et al. Liver transplantation for controversial indications: alcoholic liver disease, hepatic cancers and viral hepatitis. Transplant Proc 1991;23:1915-16.

25 Weisner RH, Lombardero $M$, and LTD Steering Committee. Long-term results of liver transplantation in adults with end-stage alcoholic liver disease [abstract]. Hepatology 1996;24:244A.

26 Bharadhwaj G, Everson O, Bilir B, et al. Relapse to alcohol consumption in liver transplant recipients followed for more than one year: early analysis of a comprehensive psychosocial evaluation [abstract]. Hepatology 1996;24:244A

27 Foster PF, Fabrega F, Sankary HN, et al. Prediction of abstinence in alcoholic liver allograft recipients [abstract]. Hepatology 1996;24:244A.

28 Neuberger JM, Tang H. Relapse after transplantation: European studies. Liver Transplantation and Surgery 1997;3: $275-9$
29 Padbury RTA, Gunson BK, Dousset B, et al. Steroid withdrawal from long-term immunosuppression in liver allograft recipients. Transplantation 1993;55:789-94.

30 Baddour N, Demetris AJ, Shah G, et al. The prevalence, rate of onset and spectrum of histological liver disease in alcohol abusing liver allograft recipients [abstract]. Gastroenterology 1992;102:A779.

31 Candinas D, Gunson BK, Nightingale P, et al. Sex mismatch as a risk factor for chronic rejection of liver allogafts. Lancet 1995;346:1117-21.

32 Radosavljevic M, Temsch E, Hammer J, et al. Elevated levels of serum carbohydrate deficient transferrin are not specific for alcohol abuse in patients with liver disease. $\mathcal{F}$ Hepatol 1995;23:706-11.

33 Anand AC, Ferraz-Neto BH, Nightingale P, et al. Liver transplantation for alcoholic liver disease: evaluation of a selection protocol. Hepatology 1997;25:1478-84.

34 Foster PF, Fabrega F, Karademir S, et al. Predication of abstinence from ethanol in alcoholic recipients following liver transplantation. Hepatology 1997;25:1469-77.

35 Sherlock S. Alcoholic liver disease. Lancet 1995;345:227-9.

36 Bernard PH, Le Bail B, Carles J, et al. Liver retransplantation for alcoholic cirrhosis recurring within a 21 -month period. Transplant Int 1996;9:524-5. 